Z Gerontol Geriat 2012 $\cdot 45: 260-261$

DOI 10.1007/s00391-012-0330-6

Online publiziert: 24. Mai 2012

(c) Springer-Verlag 2012

\section{W. von Renteln-Kruse ${ }^{1} \cdot$ T. Nikolaus ${ }^{2}$}

${ }^{1}$ Zentrum für Geriatrie und Gerontologie, Albertinen-Haus,

Wissenschaftliche Einrichtung an der Universität Hamburg

${ }^{2}$ AGAPLESION Bethesda Klinik Ulm, Akademisches Krankenhaus der Universität Ulm

\title{
Gesundes Altwerden
}

Spezifische Behandlungserfordernisse älterer Menschen rücken zunehmend auch in die breitere öffentliche Wahrnehmung [1]. Hierzu zählen zu Recht Programme mit der Zielrichtung aktiver Gesunderhaltung/-förderung im Alter sowie Maßnahmen der Prävention. Neben der Verhütung von Krankheiten besitzt Prävention im Alter einen Bezug zur zentralen Frage, ob die Entwicklung von Hilfs- und Pflegebedürftigkeit bzw. von Frailty positiv beeinflussbar ist [2].

Wird eine positive Beeinflussung angenommen, ist die frühzeitige Erkennung sich abzeichnender Verluste funktionaler Kompetenz älter werdender Menschen sinnvoll. Dies gilt besonders dann, wenn angenommen wird, dass es sich um einen dynamischen Prozess mit Übergangsphasen handelt [3]. Verschiedene Kriterien zur Bestimmung von Frailty wurden bisher verwendet, von denen sich einige auch als prädiktiv für z. B. Abhängigkeit und Tod erwiesen. Die Mehrzahl prädiktiv verwendbarer Parameter ist praktisch aufwendig, weshalb nach einfachen Verfahren gesucht wird.

Die Arbeit von Dapp et al. stellt ein Fragenset zum Selbstausfüllen zur Erkennung von Frailty vor, das im LUCAS-Verbundprojekt anhand der Langzeitkohorte entwickelt und überprüft wurde. In deutlichem Unterschied zu anderen Ansätzen berücksichtigt dieser Fragenset sowohl Risiken als auch Ressourcen älterer Menschen. Er besteht aus 6 LUCAS-Risikomarkern und 6 LUCAS-Reservemarkern und klassifiziert in die Gruppen FIT, preFRAIL und FRAIL. Diese Klassifizierung erwies sich als prädiktiv für die Entwicklung von Pflegebedürftigkeit und Tod. Damit liegt ein einfacher, selbst auszufüllender Fragebogen vor, der frühzeitig begin- nende Funktionsverluste anzeigt. Für ein Screening könnte dieser Fragebogen z. B. in Hausarztpraxen verwendet werden.

Ebenfalls aus dem LUCAS-Verbundprojekt stammt der Beitrag von Anders et al. zu Ergebnissen der Erweiterten Gerontologisch-Geriatrischen Assessments (EGGA). Untersucht wurden drei gemäß den Ergebnissen der o. g. Selbstauskunft klassifizierte Personengruppen. Diese unterschieden sich bezüglich Komorbidität und Medikamentengebrauch, Mobilität und Sturzrisiko, Haushaltsführung sowie Nutzung sozialer Unterstützung, jedoch nicht im Ernährungsstatus. Besonders deutlich unterschieden sich die Stichproben FIT, pre-FRAIL und FRAIL funktional durch ungewohnte Erschöpfung bzw. Müdigkeit nach alltäglicher Fortbewegung. Die Ergebnisse stützen die Erfordernis differenziert mehrdimensionaler Betrachtung von Gesundheit im Alter. Für mögliche, auch präventive Interventionen sollte die tatsächliche Handlungsfähigkeit älterer Menschen berücksichtigt werden [4].

In den letzten Jahren sind zahlreiche Studien erschienen, die gezeigt haben, wie eng der Zusammenhang zwischen körperlicher Aktivität, körperlichem Training und dem psychischen Status bei älteren Menschen ist. In einer Übersichtsarbeit fassen Gogulla, Lemke u. Hauer den derzeitigen Stand epidemiologischer Studien und randomisiert-kontrollierter Untersuchungen zusammen und geben einen Ausblick auf zukünftige Forschungsperspektiven. In der Mehrzahl der zitierten Untersuchungen zeigen sich kausale Effekte von körperlichem Training auf die Reduktion von Sturzangst und Depression.

Einen anderen Aspekt beleuchtet der Beitrag von Denkinger et al. Die Auto- ren analysierten in der Studie ActiFE Ulm (Activity and Function in the Elderly in Ulm), einer repräsentativen Bevölkerungsstudie älterer Menschen zwischen 65 und 90 Jahren, das Ausmaß der Inanspruchnahme von medizinischen Leistungen, die als Surrogatparameter für gesundes Altwerden benutzt werden kann. In die Untersuchung wurden insgesamt 1500 Probanden eingeschlossen, bei denen neben einem umfassenden geriatrischen Assessment die körperliche Aktivität mittels Akzelerometer sowie die Komorbidität, Medikation, Selbsthilfefähigkeit, körperliches und psychisches Wohlbefinden, die Inanspruchnahme medizinischer Leistungen, soziodemographische Faktoren und andere Parameter erfasst wurden. Verminderte körperliche Aktivität, selbst beurteilter Gesundheitszustand, Komorbidität, Body-Mass-Index und männliches Geschlecht zeigten sich als die besten Prädiktoren der Inanspruchnahme medizinischer Leistungen. Als Faktor, der sich am einfachsten modifizieren lässt und Möglichkeiten der Intervention bietet, stellte sich dabei die körperliche Aktivität heraus.

Bei der Überlegung zu gesundheitserhaltenden Maßnahmen wird der Fokus in der Regel auf den Patienten gelegt, pflegende Angehörige werden jedoch häufig vernachlässigt. Handelt es sich dabei um die Lebenspartner, weisen die pflegenden Angehörigen häufig ein ähnliches Alter wie die Patienten auf und leiden unter Umständen ebenfalls unter chronischen Erkrankungen. Sind die pflegenden Angehörigen hingegen Kinder der Patienten, befinden sich diese häufig in einer sehr belastenden sog. Sandwichsituation mit Erziehung der eigenen Kinder und Pflege eines Elternteils. Maßnahmen zur Ge- 
sunderhaltung und Entlastung pflegender Angehöriger stellen daher einen wichtigen Bereich in unserer Gesundheitsfürsorge dar und sollten mehr in den Fokus von wissenschaftlichen Untersuchungen rücken.

Ein Ansatz in diese Richtung ist das Ulmer Leuchtturmprojekt Demenz (ULTDEM), bei dem geprüft wurde, inwieweit durch ein besseres Case-Management $\mathrm{Ge}$ sunderhaltung und Entlastung pflegender Angehöriger von Demenzkranken erreicht werden können. In der Studie von Lukas et al. wurde versucht, durch eine einmalige Initialberatung mit Vermittlung in ein bestehendes Versorgungsnetz im Raum Ulm eine Entlastung der pflegenden Angehörigen zu erreichen. Bei der mit großem logistischen Aufwand durchgeführten Studie zeigte sich jedoch, dass ein solches Angebot nur unzureichend von den betroffenen Angehörigen wahrgenommen wird und andere Wege gesucht werden müssen, um die Angehörigen zu erreichen und die bereits bestehenden Angebote zu ihrer Entlastung bekannt zu machen und zu vermitteln.

Das Thema „Gesundes Altwerden“ ist sehr vielschichtig und weist viele Facetten auf, die bisher nur teilweise in ihren Zusammenhängen erkannt worden sind. Weitere bevölkerungsbasierte Längsschnittsstudien sind daher notwendig, um das komplexe Zusammenspiel zwischen körperlichen, psychischen und sozialen Faktoren zum gesunden Altwerden zu beleuchten, mit dem Ziel, die behinderungsfreie Lebenszeit weiter auszudehnen. Um es in Anlehnung an Gorch Fock zu sagen: „Man kann sein Leben nicht verlängern, noch verbreitern, nur vertiefen.“

W. von Renteln-Kruse

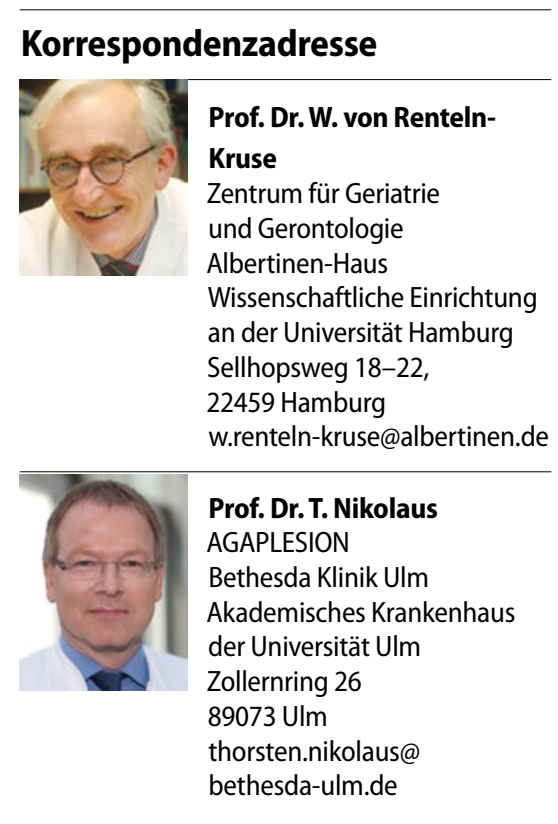

\section{Literatur}

1. Lübke N (2012) Brauchen alte Menschen eine andere Medizin? Medizinische Einordnung spezieller Behandlungserfordernisse älterer Menschen. In: Günster C, Klose J, Schmacke N (Hrsg) Versorgungs-Report 2012. Schattauer, Stuttgart, S 51-66

2. Xue Q-L (2011) The frailty syndrome: definition and natural history. Clin Geriatr Med 27:1-15

3. Michel JP, Lang P-O, Zekry D (2008) The frailty process: update of the phenotype and preventive strategies. Ann Gérontol 1:e1-e7

4. Sarkisian CA, Gruenewald TL, Boscardin WJ, Seemann TE (2008) Preliminary evidence for subdimensions of geriatric frailty: the MacArthur Study of Successful Aging. J Am Geriatr Soc 56:22922297

T. Nikolaus 